\title{
Orthopedic oncology: What's new in 2019?
}

\author{
P. Simon ${ }^{1}$
}

Published online: 15 November 2019

๑) Springer-Verlag France SAS, part of Springer Nature 2019

In countries with advanced health care networks, orthopedic oncology is a well-defined subspeciality in orthopedics. Specialized teams are in charge of early accurate diagnosis, multidisciplinary therapeutic decision including optimal surgical treatment and adjuvant therapies but also of orthopedic residents' training. Due to rarity of bone tumors, even in non-malignant tumors, an expertise is necessary. This is why an oncology training should be mandatory for all orthopedic residents. We need also evidence-based guidelines about multimodality therapies, modern surgical techniques and follow-up surveillance strategies [1].

In this special issue, three papers are dedicated to giant cell tumors. These non-malignant but aggressive tumors are often treated by non-specialized surgeons.

Tsukamoto et al. [2] studied the results of primary curettage performed by either surgeons part of a tertiary tumor center and exposed to high case volume, or surgeons not part of a specific orthopedic oncology team nor exposed to high case volume and technical training in oncology. $\mathrm{He}$ showed that adequate extensive intralesional curettage, as published in review papers or textbooks, is more important than oncology training or surgical management by specialized teams. He also showed that this first surgery following established standards can be effectively performed by earlycarrier orthopedic surgeons.

Abuhejleh et al. [3] studied the results of giant cell tumors of the distal radius, comparing intralesional curettage versus resection arthrodesis. Based on 57 cases' study from four specialized centers, he claimed for extensive intralesional curettage as this technique presents the advantage of preserving distal radius and wrist function but with a higher risk of local recurrence.

Xara-Leite et al. [4] explored the efficacy of denosumab in treating giant cell tumors of the spine in a case report and literature review. As osteoclast-like multinucleated giant

\footnotetext{
P. Simon

patrick@simon-bertrand.com

1 Lyon, France
}

cells express receptor activator of nuclear factor-kappa B (RANK), among a sea of mononuclear stromal cells that express RANK ligand (RANKL), which plays a key role in osteoclast activation, the monoclonal antibody denosumab, a RANKL specific inhibitor, prevents their pairing, therefore reducing giant cell tumor-induced bone destruction. Denosumab has been gaining an increasingly important role in recent years for treating bone or spine bone giant cell tumors, allowing significantly less morbid surgical procedures as well as successful long-term management of unresectable masses. A potential role as curative treatment has been suggested such as in his case.

Alfaro et al. [5] studied enchondromas and atypical cartilaginous tumors of the pelvis. Enchondroma is a benign tumor as atypical cartilaginous tumor is a low-grade chondrosarcoma. Both tumors exhibit similar cellular features such as mild atypia, occasional double nuclei and rare mitotic figures. So clinical and radiological data such as age, size and endosteal scalloping are useful for differential diagnosis. If risk factors for local recurrence from atypical cartilaginous tumor and enchondroma of long bones have been previously studied [6], this is the first study for pelvic tumors. The author showed in this study that atypical cartilaginous tumors can be safely treated with curettage.

Blaz Mavcic et al. published the results of a large national cohort of bone sarcomas [7]. He studied the oncological results and the endoprosthetic outcomes in a Slovenian cohort of 160 cases treated from 01/01/09 to 31/12/18. From 160 cases, 141 were treated surgically. Cumulative endoprosthetic infection rate was $12.5 \%$ when survival at $5 / 10$ years was $61 / 54 \%$ respectively.

Igoumenou et al. [8] proposed an extensive review of complications in spine surgery for metastasis. As the metastatic spinal disease is a part of a systemic disease, its optimal management requires a multidisciplinary therapeutic approach involving medical and radiation oncologists, diagnostic and interventional radiologists, neurologists, surgeons and palliative care pain control specialists. Surgery should be planned only after a thorough evaluation as proposed by Tokuhashi [9]. 
As an editor of this special tumor issue, I hope that these papers bring some new information to all orthopedic surgeons, even not well trained in oncology.

\section{Compliance with ethical standards}

Conflict of interest I have no conflict of interest to declare in relation to this paper.

\section{References}

1. Puri A (2014) Orthopedic oncology—the challenges ahead. Front Surg 1:27. https://doi.org/10.3389/fsurg.2014.00027

2. Tsukamoto S, Mavrogenis A, Tanzi P, Leone G, Akahane M, Tanaka Y, Errani C (2019) Curettage as first surgery for bone giant cell tumor: adequate surgery is more important than oncology training or surgical management by speciqlized teams. Eur J Orthop Surg Traumatol. https://doi.org/10.1007/s00590-01902535-y

3. Abuhejleh H, Wunder J, Ferguson P, Isler M, Mottard S, Werier J, Griffin A, Turcotte R (2019) Extended intralesional curettage preferred over resection-arthrodesis for giant cell tumor of the distal radius. Eur J Orthop Surg Traumatol. https://doi.org/10.1007/ s00590-019-02496-2

4. Xara-Leite F, Coutinho L, Fleming C, Magalhaes M, Oilveira V, Rodrigues-Pinto R, Cardoso P (2019) Can Denosumab cure giant cell tumors of the spine? Eur J Orthop Surg Traumatol, Case report and literature review. https://doi.org/10.1007/s0059 0-019-02554-9

5. Alfaro P, Ciani G, Herrera CA, Donati DM, Errani C (2019) Differential diagnosis and treatment of enchondromas and atypical cartilaginous tumors of the pelvis: analysis of 21 patients. Eur J Orthop Surg Traumatol. https://doi.org/10.1007/s00590-01902547-8

6. Errani C, Tsukamoto S, Ciani G et al (2017) Risk factors for local recurrence from atypical cartilaginous tumor and enchondroma of the long bones. Eur J Orthop Surg Traumatol 27:805-811. https:// doi.org/10.1007/s00590-017-1970-4

7. Mavcic B, Spiler M, Martincic D (2019) Oncologic and endoprosthetic outcomes of bone sarcoma patients. Eur J Orthop Surg Traumatol, A nationwide cohort study. https://doi.org/10.1007/ s00590-019-02503-6

8. Igoumenou VG, Mavrogenis A, Angelini A, Baracco R, Benzakour A, Benzakour T, Bork M, Vazifehdan F, Nena U, Ruggieri P (2019) Complications of spine surgery for metastasis. Eur J Orthop Surg Traumatol. https://doi.org/10.1007/s00590-01902541-0

9. Tokuhashi Y, Matsuzaki H, Oda H, Oshima M, Ryu J (2005) A revised scoring system for preoperative evaluation of metastatic spine tumor prognosis. Spine 30:2186-2191

Publisher's Note Springer Nature remains neutral with regard to jurisdictional claims in published maps and institutional affiliations. 